attacks began in infancy, at age 4 years, and at 10 years. Complex partial seizures occurred in 2 patients with onset at 14 and 25 years, and focal clonic seizures in 1 beginning at 2 years. EEGs and IQ tests were normal, and pressure-to-laugh sensations were not controlled by anticonvulsants. MRIs revealed the small hypothalamic hamartomas. (Sturm JW, Andermann F, Berkovic SF. "Pressure to laugh": An unusual epileptic symptom associated with small hypothalamic hamartomas. Neurology February 2000;54:971-973). (Reprints: Dr Samuel F Berkovic, Department of Neurology, Austin Medical Center, Studley Road, Heidelberg (Melbourne), Victoria 3084, Australia).

COMMENT. The childhood epileptic syndrome of early-onset gelastic seizures, hypothalamic hamartoma, and precocious puberty is usually associated with a poor prognosis, leading to cognitive deterioration, but in a recent report of 9 cases, 4 were cryptogenic and the outcome was more benign (see Ped Neur Briefs March 1999;13:19-20). The present authors consider their syndrome as a mild form of gelastic epilepsy.

Gelastic seizures with a frontal lobe focus. (Biraben A, Sartori E, Taussig D et al. Epileptic Disorders Dec 1999;1:221-227). A 5-year-old boy had daily episodes of forced laughter, without feelings of mirth, and with loss of contact, automatisms, facial flushing, and right facial jerking. Ictal EEG showed right frontal spikes and slowing that spread to the temporal region. MRI was normal, but interictal SPECT showed hypoperfusion in the right frontal lobe. Seizures were controlled with sodium valproate. Gelastic seizures originating in the frontal lobe are unusual, the more common locus being the diencephalon or temporal lobe.

\title{
GENETICS OF CHILDHOOD EPILEPSY
}

Genetic epilepsies are classified according to the mechanism of inheritance in three major groups: 1) Mendelian idiopathic epilepsies; 2) Non-Mendelian or "complex" epilepsies; and 3) Chromosomal disorders. Mendelian epilepsies include the autosomal dominant, benign familial neonatal and infantile convulsions, nocturnal frontal lobe epilepsy, and generalized epilepsy with febrile seizures. Non-Mendelian "complex" idiopathic epilepsies include juvenile myoclonic, febrile convulsions, childhood absence, juvenile absence, and benign epilepsy with centrotemporal spikes. Among symptomatic epilepsies, progressive myoclonic types account for $1 \%$ of childhood epilepsies. These are Mendelian (Unverricht-Lundborg, neuronal ceroid lipofuscinoses, and Lafora disease), and Non-Mendelian "complex" progressive myoclonic, associated with mitochondrial disorders. New classifications based on molecular genetics require identification of common DNA sequence variations between individuals. The authors predict that DNA from a buccal swab will be analyzed for common mutations in ion channel genes, and an antiepilepsy drug designed for the specific electrophysiological dysfunction. With the precise molecular diagnosis of the future, the EEG could become a redundant investigation. (Robinson R, Gardiner M. Genetics of childhood epilepsy. Arch Dis Childhood February 2000;82:121-125). (Respond: Dr Robinson, Department of Paediatrics, Royal Free and University College Medical School, University Street, London WC1E 6JJ, UK. - email: robert.robinson@ucl.ac.uk).

COMMENT. The epilepsies are heterogeneous in manifestations and causes. A genetic etiology may be present in more than $40 \%$ of childhood patients. 